\title{
Metamaterial hyperlens for the MIR
}

Hayashi, J. G.; Lwin, R.; Stefani, Alessio ; Fleming, S.; Argyros, A.; Kuhlmey, B. T.

\section{Published in:}

Proceedings of 41st International Conference on Infrared, Millimeter and Terahertz Waves

Link to article, DOI:

10.1109/IRMMW-THz.2016.7758986

Publication date:

2016

Document Version

Peer reviewed version

Link back to DTU Orbit

Citation (APA):

Hayashi, J. G., Lwin, R., Stefani, A., Fleming, S., Argyros, A., \& Kuhlmey, B. T. (2016). Metamaterial hyperlens for the MIR. In Proceedings of 41st International Conference on Infrared, Millimeter and Terahertz Waves [7758986] IEEE. International Conference on Infrared, Millimeter and Terahertz Waves https://doi.org/10.1109/IRMMW-THz.2016.7758986

\section{General rights}

Copyright and moral rights for the publications made accessible in the public portal are retained by the authors and/or other copyright owners and it is a condition of accessing publications that users recognise and abide by the legal requirements associated with these rights.

- Users may download and print one copy of any publication from the public portal for the purpose of private study or research.

- You may not further distribute the material or use it for any profit-making activity or commercial gain

- You may freely distribute the URL identifying the publication in the public portal 


\title{
Metamaterial hyperlens for the MIR
}

\author{
J. G. Hayashi*, R. Lwin*, A. Stefani*‡, S. Fleming*, A. Argyros*, and B. T. Kuhlmey*† \\ *Institute of Photonics and Optical Science (IPOS), School of Physics, University of Sydney, NSW, Australia \\ E-mail: juliano.hayashi@sydney.edu.au \\ ${ }^{\dagger}$ Centre for Ultrahigh bandwidth Devices for Optical Systems (CUDOS), \\ School of Physics, University of Sydney, NSW, Australia \\ $\ddagger$ DTU Fotonik, Department of Photonics Engineering, \\ Technical University of Denmark, DK-2800 Kgs. Lyngby, Denmark
}

\begin{abstract}
We report the first metamaterial tapered hyperlens for operation in the mid-infrared $(3 \mu \mathrm{m})$ made of 470 tin wires embedded in soda-lime glass. The taper presents a magnification of $2 x$ and is $62.5 \mu \mathrm{m}$ in length. The structure on the smallest side has an average wire diameter and spacing of 300 and $600 \mathrm{~nm}$, respectively. Preliminary modeling of the hyperlens indicates that the overall loss for the high spatial frequency modes in such a device is smaller than $36 \mathrm{~dB}$, making sub-diffraction imaging feasible.
\end{abstract}

\section{INTRODUCTION AND BACKGROUND}

$\mathbf{I}$ $\mathrm{N}$ hyperbolic media such as wire arrays [1], the hyperbolic dispersion allows the propagation of high spatial frequencies, that would be evanescent in isotropic media. A tapered hyperlens magnifies such frequencies, allowing subdiffraction imaging in the far field [2]. Recently, our group has demonstrated a wire array metamaterial fiber for the $\mathrm{THz}$ region, made of indium wires embedded in PMMA/Zeonex [3]. However, due to the rheological properties of these materials, the Plateau-Rayleigh instability [4] limits the wire diameter to a few microns [5], which makes impractical the fabrication of a sub-wavelength structure for the mid-infrared using this system. Such sub-wavelength structure fabrication is possible by replacing the polymer with soft-glasses, which decreases the surface tension between the molten metal and the dielectric during the drawing, shifting down the PlateauRayleigh instability limit. In the present work, we demonstrate the fabrication of the first wire array metamaterial hyperlens for operation at a wavelength $\lambda=3 \mu \mathrm{m}$, using soda-lime glass as the dielectric host and achieving a wire array structure with an average wire diameter $\left(d_{\mathrm{avg}}\right)$ and spacing $(\Lambda)$ around a few hundreds of $\mathrm{nm}$. In addition, we also present preliminary modeling of the high spatial frequencies mode losses in such a structure.

\section{RESULTS}

The wire array metamaterial preform based on tin and sodalime glass was fabricated by the fiber drawing technique, then tapered to a hyperlens with a commercial pipette puller ( $\mathrm{P}$ 97, Sutter Instrument). Figure 1(a) shows the drawn preform with outside diameter of $300 \mu \mathrm{m}, d_{\mathrm{avg}}=3 \mu \mathrm{m}$ and $\Lambda=6 \mu \mathrm{m}$. Figure 1(b) shows a side-on optical micrograph of the resultant taper, where the horizontal lines define the hyperlens, with outside diameters around $30 \mu \mathrm{m}\left(d_{\mathrm{avg}}=300 \mathrm{~nm}, \Lambda=600 \mathrm{~nm}\right)$ and $60 \mu \mathrm{m}\left(d_{\mathrm{avg}}=600 \mathrm{~nm}, \Lambda=1200 \mathrm{~nm}\right)$, respectively. In order to obtain an initial estimate of the loss of the high spatial frequency modes in such a tapered hyperlens, we numerically modeled the quasi-TEM mode in the indefinite tin/soda-lime wire array structure with COMSOL [6], for different structures with a fixed $d / \Lambda$, emulating the taper in different cross section regions along length. Figure 1(c) shows the loss in $\mathrm{dB} / \mu \mathrm{m}$ for the quasi-TEM mode with three different spatial frequencies as a function of $d$, with $d / \Lambda=0.5$, at $\lambda=3 \mu \mathrm{m}$, where $k_{\perp} \max$ corresponds to the transverse wave vector component matching the edge of the first Brillouin zone, which gives the maximum resolution.
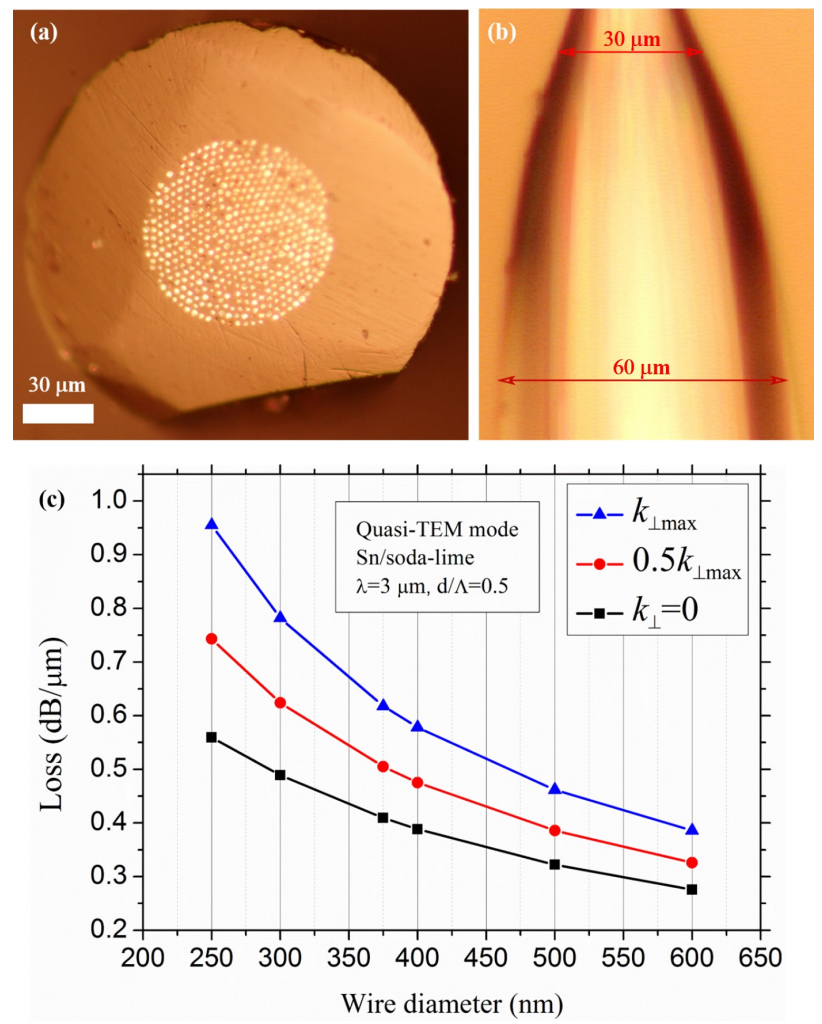

Fig. 1. (a) Drawn metamaterial preform (tin/soda-lime) with outside diameter of $300 \mu \mathrm{m}\left(d_{\mathrm{avg}}=3 \mu \mathrm{m}\right.$ and $\left.\Lambda=6 \mu \mathrm{m}\right)$. (b) Tapered hyperlens side image. (c) Loss in $\mathrm{dB} / \mu \mathrm{m}$ for the indefinite wire array structure (tin/soda-lime) with $d / \Lambda=0.5$, as a function of $d$, at $\lambda=3 \mu \mathrm{m}$. The black, red and blue curves represent the modes with transverse wave vector equal to $0,0.5 k_{\perp} \max$, and $k_{\perp} \max$, respectively. 
Using the losses presented in Fig. 1(c) and discretizing our hyperlens (Fig. 1(b)) in several slices of $5 \mu \mathrm{m}$ along length, we estimate that the quasi-TEM mode with $k_{\perp} \max$ will present a loss smaller than $36 \mathrm{~dB}$, making sub-diffraction imaging feasible in such a device.

In the near future, we intend to image in the far field a diffraction limited double aperture fabricated on the smallest side of the hyperlens using sputtering gold deposition and FIB milling.

\section{CONCLUSION}

We have fabricated the first wire array metamaterial tapered hyperlens for operation at $\lambda=3 \mu \mathrm{m}$, with a magnification factor of 2. The analyses of the numerical losses obtained by our simulations and the hyperlens profile indicate that the presented device will have an overall loss smaller than $36 \mathrm{~dB}$ for the mode with $k_{\perp} \max$, making sub-diffraction imaging feasible. A full study regarding the taper fabrication parameters and their influence on the hyperlens profile and on the wire array structure quality is under development. This fabrication optimization can lead to an improvement of the magnification factor and of the overall loss of the final device. The initial probe shows a relatively uniform wire array structure along the taper when the hyperlens is fabricated under high tension. Preliminary results will be presented at the conference, including cross section SEM pictures of both sides of the hyperlens, the fabrication of the diffraction limited double aperture and the initial results of its imaging through the device.

\section{ACKNOWLEDGEMENT}

This research was supported by the Australian Research Council (ARC) under the Discovery Project scheme number DP120103942 and DP140104116, and it was performed in part at the Optofab node of the Australian National Fabrication Facility (ANFF) using Commonwealth and NSW State Government funding.

The author Juliano Hayashi would like to thank the Science without Borders Program by CAPES (Brazil) for the financial support under grant 9468/13-7.

A.S. acknowledges the Eugen Lommel Stipend for financial support. This project has received funding from the European Unions Horizon 2020 research and innovation programme under the Marie Sklodowska-Curie grant agreement No 708860.

\section{REFERENCES}

[1] P. A. Belov, G. K. Palikaras, Y. Zhao, A. Rahman, C. R. Simovski, Y. Hao, and C. Parini, "Experimental demonstration of multiwire endoscopes capable of manipulating near-fields with subwavelength resolution," Appl. Phys. Lett. 97(19), 191905 (2010).

[2] Z. Jacob, L. V. Alekseyev, and E. Narimanov, "Optical Hyperlens: Farfield imaging beyond the diffraction limit," Opt. Express 14, 8247-8256, 2006.

[3] A. Tuniz, K. J. Kaltenecker, B. M. Fischer, M. Walther, S. C. Fleming, A. Argyros, and B. T. Kuhlmey, "Metamaterial fibres for subdiffraction imaging and focusing at terahertz frequencies over optically long distances", Nat. Commun. 4, 2706, 2013.

[4] S. Tomotika, "On the instability of a cylindrical thread of a viscous liquid surrounded by another viscous fluid," Proc. R. Soc. Lon. A Math. 150(870), 322337 (1935).
[5] O. T. Naman, M. R. New-Tolley, R. Lwin, A. Tuniz, A.H. Al-Janabi, I. Karatchevtseva, S. C. Fleming, B. T. Kuhlmey, and A. Argyros, "Indefinite media based on wire array metamaterials for $\mathrm{THz}$ and MidIR,” Adv. Opt. Mater. 1(12), 971977, 2013.

[6] J. G. Hayashi, S. Fleming, B. T. Kuhlmey and A. Argyros, "Metal selection for wire array metamaterials for infrared frequencies," Opt. Express 23, 29867-29881, 2015. 